\title{
Synchronous Adenocarcinoma and Follicular Lymphoma of the Stomach
}

\author{
Masaya Iwamuro ${ }^{1,2}$, Atsushi Imagawa ${ }^{1}$, Naruyuki Kobayashi ${ }^{3}$, Yoshitsugu Kubota ${ }^{4}$, \\ Katsuya Miyatani ${ }^{5}$, Katsuyoshi Takata ${ }^{6}$ and Hiroyuki Okada ${ }^{7}$
}

\begin{abstract}
A 73-year-old Japanese man with synchronous follicular lymphoma and adenocarcinoma of the stomach underwent curative surgical resection. The follicular lymphoma lesion was preoperatively diagnosed as extranodal marginal zone lymphoma of mucosa-associated lymphoid tissue (MALT lymphoma) according to biopsy samples. However, postoperative pathological evaluations revealed components of CD10-positive and CD10-negative lymphoma cells within the lymphoma lesion. This case highlights the potential difficulty of diagnosing gastric follicular lymphoma. In such cases, conducting repeat pathological examinations of biopsy samples or resected specimens is required to obtain a correct diagnosis of follicular lymphoma.
\end{abstract}

Key words: follicular lymphoma, gastrointestinal endoscope, gastric neoplasms, gastrointestinal lymphoma, gastric cancer

(Intern Med 52: 907-912, 2013)

(DOI: 10.2169/internalmedicine.52.7948)

\section{Introduction}

The category of primary intestinal follicular lymphoma was recently established. This entity was formally cited in the latest version of the classification of lymphoid tissues issued by the World Health Organization $(1,2)$. Involvement of the duodenum is a predominant feature of primary intestinal follicular lymphoma. Recent case series from several institutions have revealed a high rate of extensive involvement of the jejunum and/or ileum, accounting for $66.7 \%$ to $100 \%$ of patients who undergo entire gastrointestinal tract surveillance with video capsule endoscopy or double balloon enteroscopy (3-8). In contrast, gastric involvement is less frequent in cases of follicular lymphoma, accounting for $2 \%$ to $29 \%$ of follicular lymphoma cases (9-12). Takata and coworkers reported that, among 125 patients with primary intestinal follicular lymphomas, involvement of the duodenum was found in 111 (89\%) cases, involvement of the jejunum was found in $50(40 \%)$ cases and involvement of the ileum was found in $28(22 \%)$ cases, whereas involvement of the stomach was seen in only two (2\%) cases (3).

In this report, we present a case of follicular lymphoma of the stomach. This patient had the additional uncommon feature of concomitant gastric adenocarcinoma. It is also noteworthy that in the preoperative pathological evaluation of this lymphoma lesion, it was difficult to reach a diagnosis of follicular lymphoma. Consequently, potential pitfalls in the diagnosis of gastric follicular lymphomas are also discussed.

\section{Case Report}

A 73-year-old Japanese man underwent an esophagogastroduodenoscopic examination at his family clinic due to fasting epigastric discomfort. The patient was diagnosed with adenocarcinoma of the stomach and referred to Mitoyo General Hospital for further investigation and treatment. He

${ }^{1}$ Department of Gastroenterology, Mitoyo General Hospital, Japan, ${ }^{2}$ Department of Gastroenterology and Hepatology, Okayama University Graduate School of Medicine, Dentistry, and Pharmaceutical Sciences, Japan, ${ }^{3}$ Department of Surgery, Sumitomo Besshi Hospital, Japan, ${ }^{4}$ Department of Transfusion Medicine, Faculty of Medicine, Kagawa University, Japan, ${ }^{5}$ Department of Pathology, Mitoyo General Hospital, Japan, ${ }^{6}$ Department of Pathology, Okayama University Graduate School of Medicine, Dentistry, and Pharmaceutical Sciences, Japan and ${ }^{7}$ Department of Endoscopy, Okayama University Hospital, Japan

Received for publication April 12, 2012; Accepted for publication January 16, 2013

Correspondence to Dr. Masaya Iwamuro, iwamuromasaya@yahoo.co.jp 

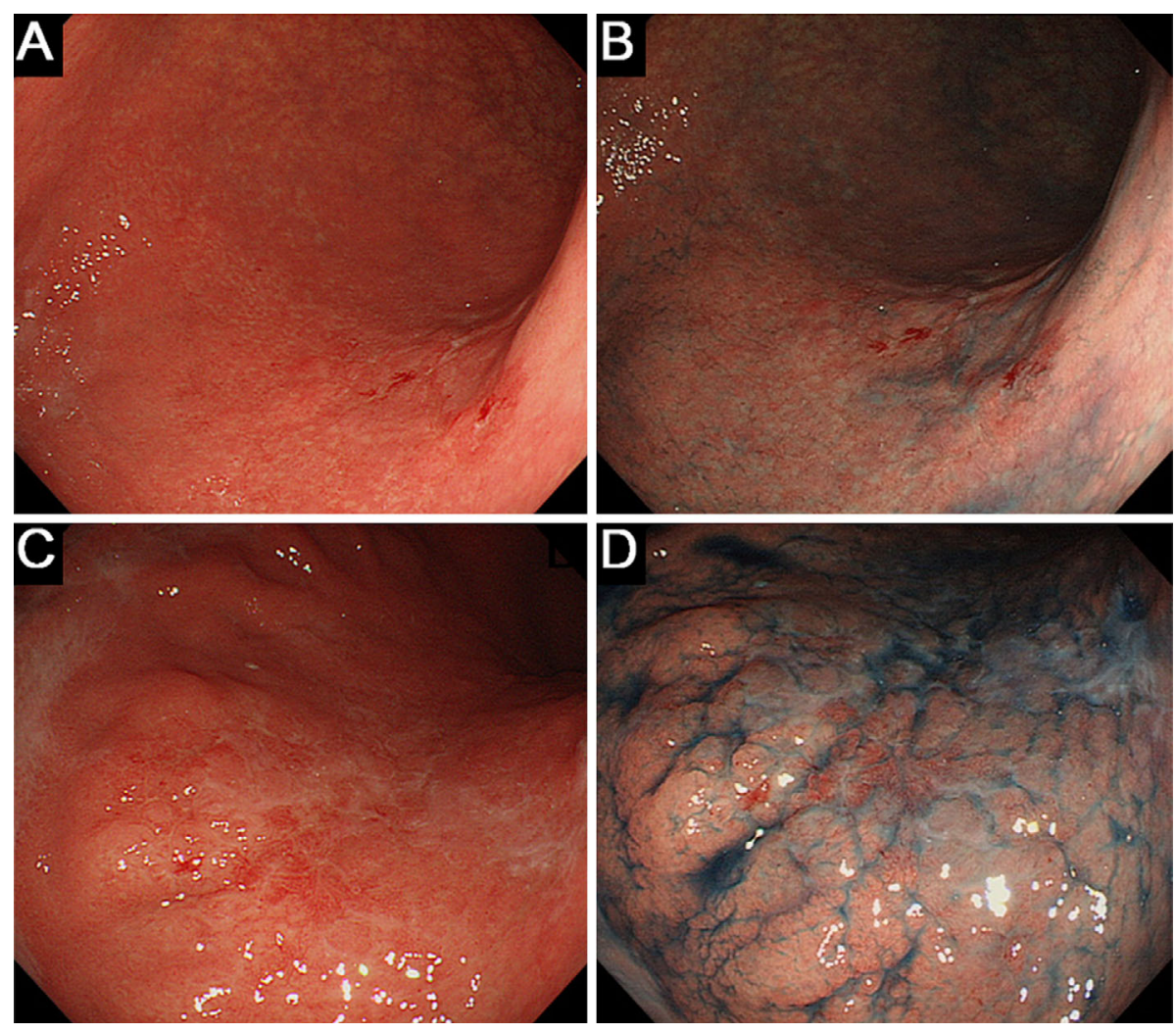

Figure 1. Esophagogastroduodenoscopic images of the stomach. A depressed lesion was seen in the posterior wall of the lower gastric body $(\mathrm{A}, \mathrm{B})$ that was diagnosed as adenocarcinoma. In the greater curvature of the upper gastric body, an irregularly shaped shallow, depressed lesion with a vague margin was seen $(\mathrm{C}, \mathrm{D})$. Although this lesion was preoperatively diagnosed as MALT lymphoma, a reevaluation of the surgically resected specimen led to a final diagnosis of follicular lymphoma.

had a previous history of benign prostate hypertrophy and chronic obstructive pulmonary disease (COPD) and had started home oxygen therapy one year previously. The FEV1 on pulmonary function tests was $61.6 \%$ of the predicted value (COPD stage II). The patient had no history of gastrointestinal disease. His weight had decreased from $72 \mathrm{~kg}$ to $53 \mathrm{~kg}$ over the previous six months. A physical examination revealed no abnormalities, and there was no evidence of hepatosplenomegaly or peripheral lymphadenopathy. All laboratory findings, including the levels of lactate dehydrogenase (LDH) and soluble interleukin-2 receptor (sIL-2R), were within the normal ranges. A second esophagogastroduodenoscopic examination showed two lesions in the stomach: a depressed lesion measuring approximately $30 \mathrm{~mm}$ in diameter on the posterior wall of the lower gastric body (Fig. 1A, B) and an irregularly shaped shallow depressed lesion with a vague margin of approximately $60 \mathrm{~mm}$ in diameter at the greater curvature of the upper gastric body (Fig. 1C, D). Histopathological examinations of the biopsied specimens obtained from the former lesion revealed well- to moderately-differentiated tubular adenocarcinoma of the stomach. The biopsied samples obtained from the latter lesion showed dense infiltration of small- to medium-sized lymphoid cells into the gastric mucosa (Fig. 2). An immunohistochemical study demonstrated the lymphoid cells to be positive for CD20 and negative for CD3 and cytokeratin AE1/AE3. Taken together, these results indicated that a neoplastic expansion of $\mathrm{B}$ cells had occurred. Based on these pathological features, synchronous adenocarcinoma and extranodal marginal zone lymphoma of mucosa-associated lymphoid tissue (MALT lymphoma) in the stomach were suspected. Contrast-enhanced computed tomography (CT) scanning of the neck, chest, abdomen and pelvis showed no other involvement, except for multiple enlarged paragastric lymph nodes.

Since the possibility of lymph node metastasis as a result of gastric carcinoma could not be excluded, total gastrectomy with lymph node excision, cholecystectomy and splenectomy was performed. In the surgically resected specimens (Fig. 3), well-differentiated tubular adenocarcinoma confined to the mucosal and submucosal layers was pathologically confirmed (Fig. 4). Helicobacter pylori was also detected in the specimens. In the other lesion, nodular neoplastic follicles composed of small- to medium-sized cleaved cells were seen (Fig. 5). These cells were located primarily in the mucosal and submucosal layers, although they had also partly infiltrated the proper muscle layer and subserosal layer. However, no lymphoepithelial lesions were detected. An immunohistochemical study revealed that the lymphoma cells forming lymphoid follicles were positive for CD20, 


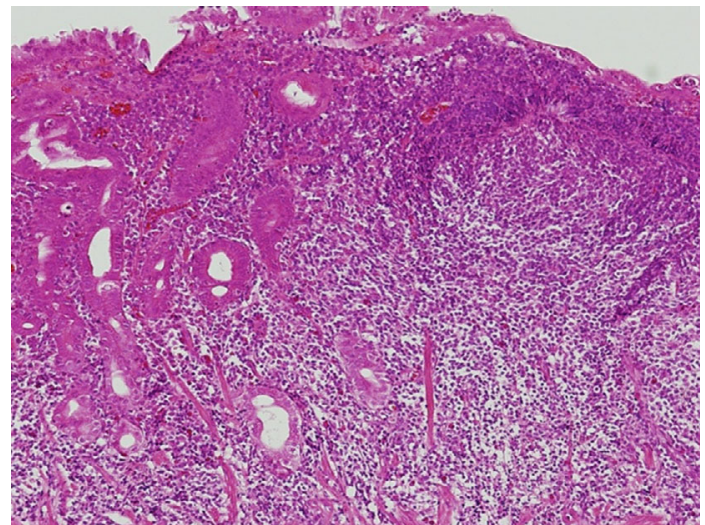

Figure 2. Histopathological examination of the biopsied specimen. In the biopsied specimen obtained from the lymphoma lesion, dense infiltration of small- to medium-sized lymphoid cells was seen. In the immunohistochemical analysis, the neoplastic cells were positive for $\mathrm{CD20}$ and negative for CD3 and cytokeratin AE1/AE3. These results indicated the neoplastic expansion of B-cells.

CD10, BCL-2 and BCL-6, yet were negative for CD3 and CD5. Accordingly, a diagnosis of follicular lymphoma of the stomach, rather than MALT lymphoma, was made. The lymphoma cells outside of the lymphoid follicles, especially those located in the surface layer of the mucosa, were largely negative for CD10. However, CD10-positive neoplastic cells were only sparsely observed (Fig. 6). Tissuefluorescence in situ hybridization (T-FISH) for $\mathrm{t}(14 ; 18)(\mathrm{q} 32$; q21)/IGH-BCL2 translocation was performed; however, no translocation was present in either the CD10-positive lymphoma cells or the CD10-negative lymphoma cells. Infiltration of lymphoma cells into the paragastric lymph nodes and spleen was also noted, whereas no adenocarcinoma components were detected in either the lymph nodes or the spleen. The postoperative diagnosis was follicular lymphoma of the stomach, Grade 1, pathological stage $\mathrm{II}_{1} \mathrm{E}$ (spleen), based on the Lugano staging system for the classification of gastrointestinal tract lymphoma $(13,14)$. A bone marrow aspirate and biopsy as well as whole-body positron emission tomography (PET) scans were postoperatively performed, and no persistence of lymphoma or carcinoma was detected. Six courses of chemotherapy comprised of rituximab plus CHOP (cyclophosphamide, doxorubicin, vincristine and prednisone) were administered, and no recurrence was detected for the 30 months following surgery.

\section{Discussion}

Simultaneous occurrence of adenocarcinoma and lymphoma in the stomach is a rare event. For example, Nakamura and co-workers previously reported that only $9 / 9,150$ $(0.098 \%)$ of cases involving gastric carcinoma also involved primary gastric lymphoma (15). Similarly, Noda and colleagues reported an incidence of 2/2,438 (0.082\%) for cases of gastric carcinoma (16). According to a review of reported

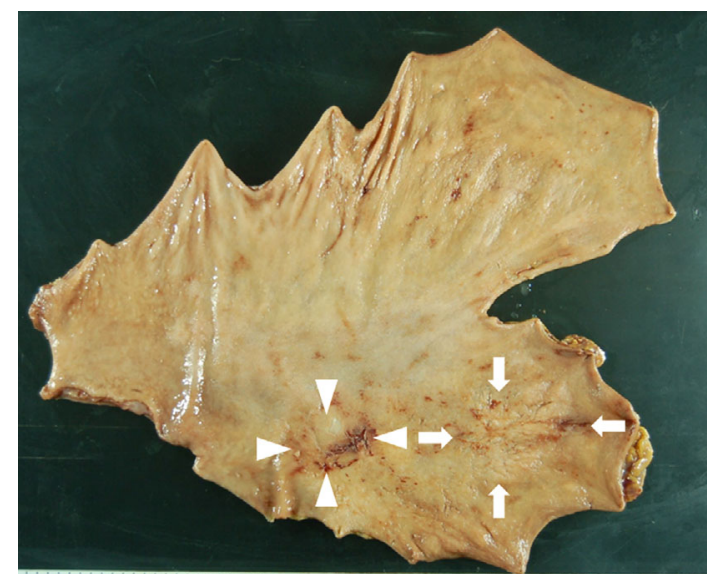

Figure 3. Macroscopic image of the surgically resected stomach. A carcinoma lesion was present in the lower gastric body (indicated with arrowheads) and a lymphoma lesion was present in the upper gastric body (indicated with arrows).

cases of synchronous gastric adenocarcinoma and lymphoma, the major lymphoma subtype is MALT lymphoma (17). It is widely accepted that Helicobacter pylori is a common carcinogen causative of both gastric adenocarcinoma and gastric MALT lymphoma (18). Therefore, it is hypothesized that Helicobacter pylori plays an important role in the synchronous occurrence of adenocarcinoma and MALT lymphoma in the stomach. Indeed, Sakai and colleagues reported a $100 \%$ positivity rate of Helicobacter pylori infection among 13 reviewed cases of synchronous adenocarcinoma and MALT lymphoma in the stomach (19). However, Helicobacter pylori may not participate in the pathogenesis of follicular lymphoma. In most reported cases of gastrointestinal follicular lymphoma, the eradication of Helicobacter pylori results in unchanged gastric lesions. Moreover, progression of gastric lesions has been observed following successful treatment of Helicobacter pylori (20-24). Taken together, these findings suggest that the neoplastic follicles of follicular lymphoma are not composed of antigen-driven cells, in contrast to those of MALT lymphoma (20). Furthermore, the incidence of synchronous gastric adenocarcinoma and gastric follicular lymphoma is quite low. Therefore, in the present case, the simultaneous detection of two distinct neoplasms appears to have been coincidental.

The macroscopic features of gastric follicular lymphoma have not been fully revealed due to the rarity of this disease. The reported morphology of gastric follicular lymphoma varies from elevated nodular lesions (25) to mucosal inflammation and ulceration (26). As common practice, the use of biopsies is essential to diagnose gastrointestinal lymphoma. In the present patient, MALT lymphoma was suspected as a preoperative diagnosis of the lymphoma lesion. Similar misinterpretations of gastrointestinal follicular lymphoma as MALT lymphoma have been described in other reports $(21,26)$. Pathologically, the major differential diagnoses of follicular lymphoma include MALT lymphoma, man- 

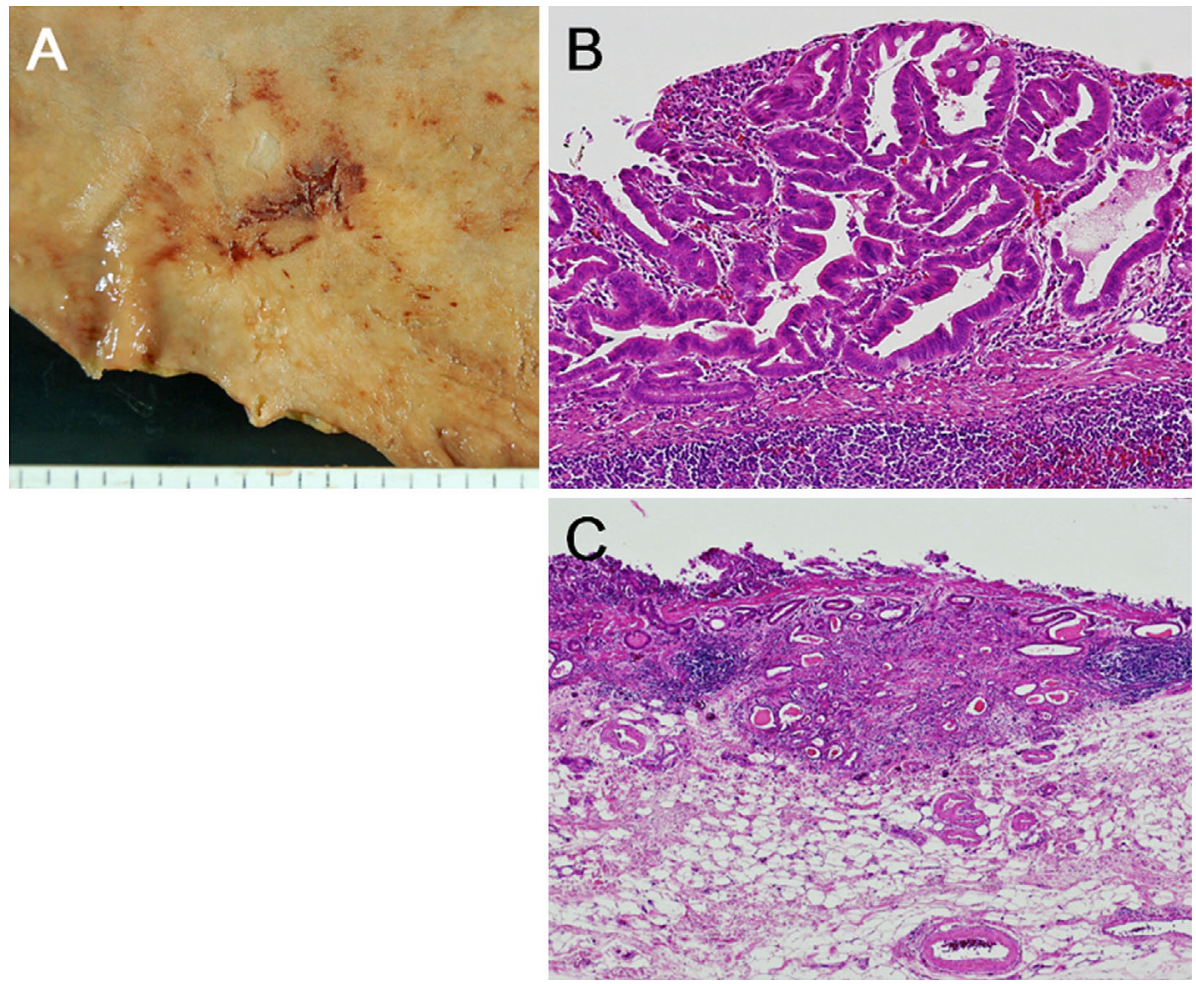

Figure 4. Macroscopic image (A) and microscopic images $(\mathrm{B}, \mathrm{C})$ of the lesion in the lower gastric body. A diagnosis of well-differentiated tubular adenocarcinoma was made. Hematoxylin and Eosin staining shows the partial infiltration of cancer cells into the submucosal layer $(C)$.
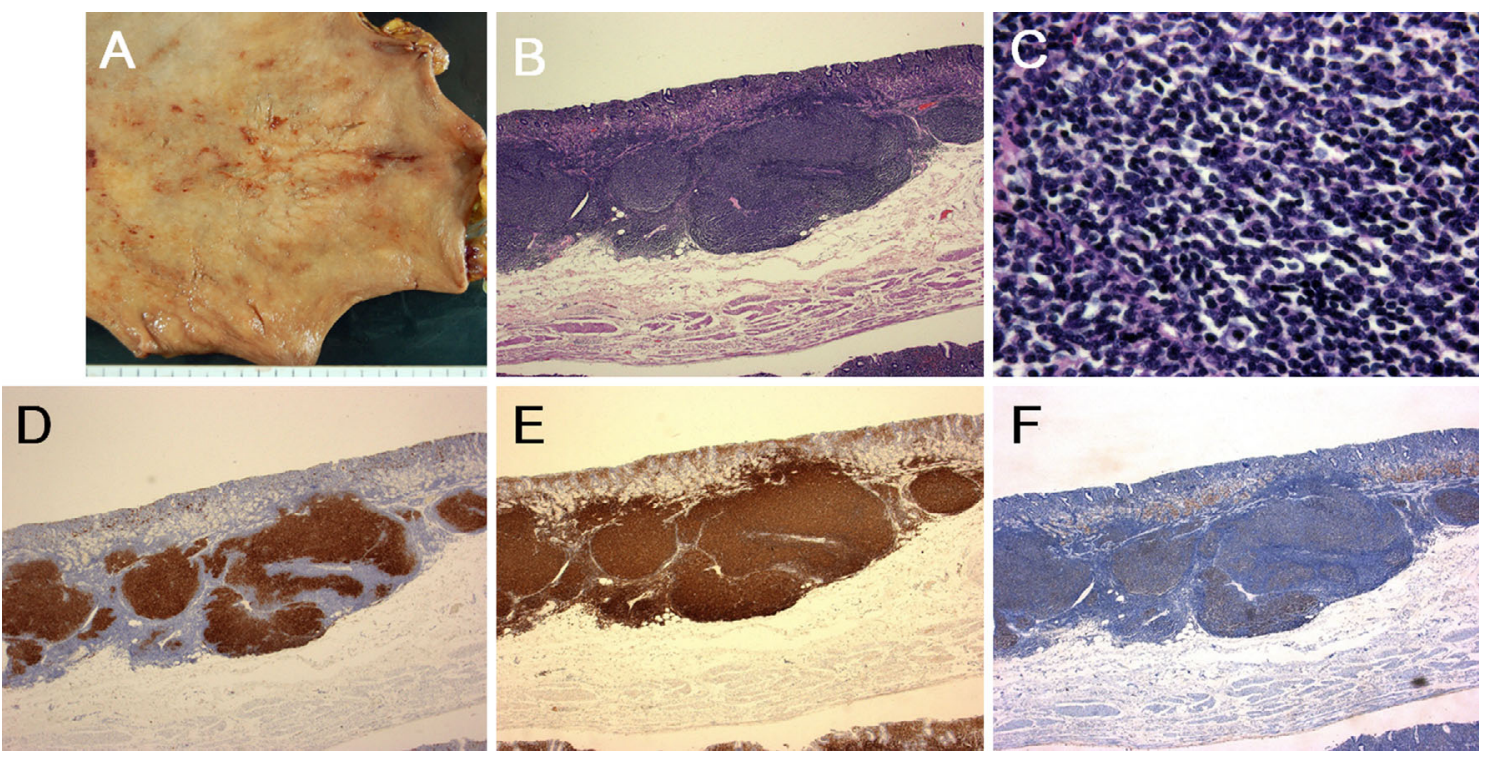

Figure 5. Macroscopic (A) and microscopic (B-E) images of the lesion in the upper gastric body. Hematoxylin and Eosin staining shows uniform, small-cleaved cells that infiltrated into the gastric wall from the mucosal layer through the subserosal layer (B, C). An immunohistochemical study revealed that most of the lymphoma cells were positive for CD10 (D), BCL-2 (E) and BCL-6 (F).

tle cell lymphoma and reactive lymphoid hyperplasia. The neoplastic cells present in low-grade B-cell lymphomas, namely, follicular lymphoma, MALT lymphoma and mantle cell lymphoma, share similar morphological features to some extent. For example, such lymphomas are primarily composed of small- to medium-sized lymphoid cells of B- cell origin. Additionally, lymphoepithelial lesions, which represent a well-known characteristic of MALT lymphomas, have occasionally been described in cases of gastric follicular lymphoma (26). Generally, subcategorizing low-grade Bcell lymphomas according to their morphological features can be difficult and typically requires immunohistochemical 

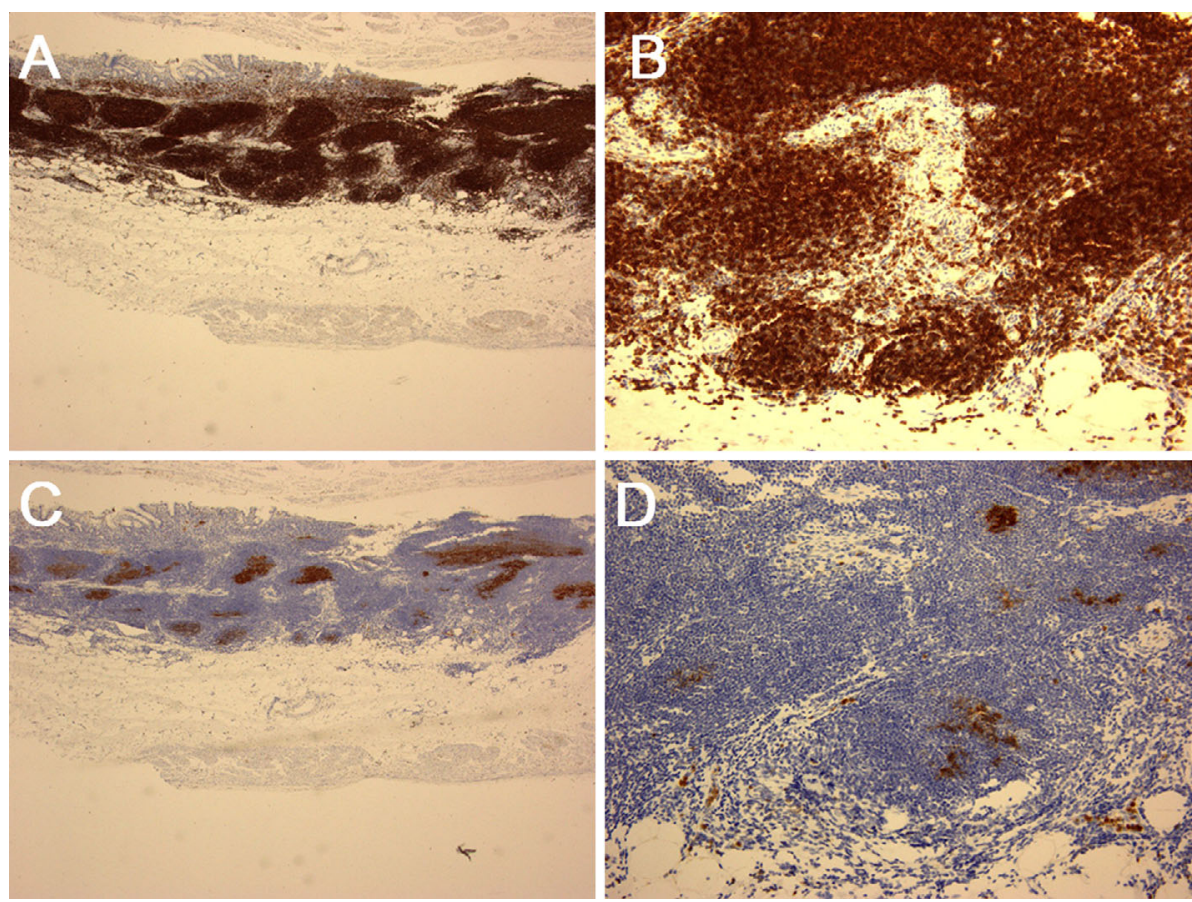

Figure 6. Immunohistochemical images of the area showing CD10-negative lymphoma cells. The infiltrating lymphoma cells were mostly positive for BCL2 expression (A, B), while subsets of infiltrating cells were found to be negative for CD10 expression, especially in the interfollicular areas $(C$, D).

staining. In mantle cell lymphoma, the lymphoma cells are positive for CD5 and cyclin D1. Typical follicular lymphoma consists of CD10-positive neoplastic lymphoid cells. In contrast, the lymphoma cells in MALT lymphoma are negative for CD10, CD5 and cyclin D1. However, as described above, most low-grade B-cell lymphomas in the stomach are MALT lymphomas, which can impede further immunohistochemical surveillance for CD10, CD5 and cyclin D1 and may lead to the misdiagnosis of the lesions as MALT lymphomas.

In the present case, the neoplastic cells present in the follicular lymphoma were partly negative for CD10 (Fig. 6). Despite the partial CD10-negativity of the lymphoma cells, the diagnosis of follicular lymphoma was supported by distinctive pathological features such as the monotonous proliferation of lymphoma cells with a strong expression of CD10 and nodular formation of neoplastic follicles (Fig. 5). A negative expression of CD10 in interfollicular infiltrating cells can be observed in typical follicular lymphoma cases (27). While previous studies have also reported that the neoplastic cells of MALT lymphomas can be positive for CD10 in rare instances $(28,29)$, these cases involved either a very weak expression of CD10 or moderately positive membrane staining for CD10. Therefore, based on the strong expression of CD10 observed in the present case, a diagnosis of MALT lymphoma with follicular colonization was excluded.

The ability to detect the $\mathrm{t}(14 ; 18)(\mathrm{q} 32 ; \mathrm{q} 21) / \mathrm{IGH}-\mathrm{BCL} 2$ translocation in CD10-negative lesions provides direct evidence for a genetic alteration that is typical of follicular lymphoma rather than MALT lymphoma. However, genetic evidence of this translocation was not available for the patient reported here. Yanai et al. reported that the $t(14 ; 18)$ (q32;q21)/IGH-BCL2 translocation was detected in 7/11 (63.6\%) gastric follicular lymphomas (12). We recently reported that the frequency of the translocation in gastric follicular lymphoma samples is significantly lower than that observed in duodenal follicular lymphomas (30). For example, this translocation was detected in only $3 / 8$ (37.5\%) gastric follicular lymphomas versus $13 / 15(86.7 \%)$ duodenal follicular lymphomas. Consequently, in the diagnosis of gastric lymphomas, a lack of the $\mathrm{t}(14 ; 18)(\mathrm{q} 32 ; \mathrm{q} 21) / \mathrm{IGH}-\mathrm{BCL} 2$ translocation does not exclude the possibility of follicular lymphoma because approximately $40-60 \%$ of all cases are negative for such translocation.

In our patient, even if CD10 staining had been performed preoperatively, it might have nonetheless been difficult to arrive at the diagnosis of follicular lymphoma due to the presence of CD10-negative lymphoma cells. This highlights two major issues associated with the immunohistochemical analysis of follicular lymphomas. First, approximately $20 \%$ of cases of follicular lymphoma are negative for CD10 (27, 31). Second, as described in our patient, neoplastic cells in follicular lymphoma can be partly negative for CD10. Such ambiguities pose diagnostic challenges, which may lead to an inaccurate diagnosis of gastrointestinal lymphomas, especially in small biopsy specimens (25). In such cases, neoplastic follicles must be identified in larger specimens or multiple biopsied specimens to arrive at the correct diagnosis of follicular lymphoma. Otherwise, such cases may be confused with MALT lymphoma.

In conclusion, a case of synchronous adenocarcinoma and 
follicular lymphoma of the stomach is reported. This case highlights the difficulty of diagnosing gastric follicular lymphoma since it rarely occurs in the stomach and because it shares pathomorphological similarities with MALT lymphoma. It is also particularly noteworthy in this case that the neoplastic cells were found to be negative for CD10. Consequently, gastroenterologists and endoscopists should be aware of such pitfalls in the pathological classification of gastrointestinal lymphomas.

The authors state that they have no Conflict of Interest (COI).

\section{References}

1. Harris NL, Swerdlow SH, Jaffe ES, et al. Follicular lymphoma. In: WHO Classification of Tumours of Haematopoietic and Lymphoid Tissues. Swerdlow S, Campo E, Harris NL, et al, Eds. IARC, Lyon, 2008: 220-226.

2. Yoshino T, Miyake K, Ichimura $\mathrm{K}$, et al. Increased incidence of follicular lymphoma in the duodenum. Am J Surg Pathol 24: 688693, 2000.

3. Takata K, Okada H, Ohmiya N, et al. Primary gastrointestinal follicular lymphoma involving the duodenal second portion is a distinct entity: a multicenter, retrospective analysis in Japan. Cancer Sci 102: 1532-1536, 2011.

4. Schmatz AI, Streubel B, Kretschmer-Chott E, et al. Primary follicular lymphoma of the duodenum is a distinct mucosal/submucosal variant of follicular lymphoma: a retrospective study of 63 cases. J Clin Oncol 29: 1445-1451, 2011.

5. Akamatsu T, Kaneko Y, Ota H, Miyabayashi H, Arakura N, Tanaka E. Usefulness of double balloon enteroscopy and video capsule endoscopy for the diagnosis and management of primary follicular lymphoma of the gastrointestinal tract in its early stages. Dig Endosc 22: 33-38, 2010.

6. Higuchi N, Sumida Y, Nakamura K, et al. Impact of doubleballoon endoscopy on the diagnosis of jejunoileal involvement in primary intestinal follicular lymphomas: a case series. Endoscopy 41: 175-178, 2009.

7. Kodama M, Kitadai Y, Shishido T, et al. Primary follicular lymphoma of the gastrointestinal tract: a retrospective case series. Endoscopy 40: 343-346, 2008.

8. Nakamura S, Matsumoto T, Umeno J, et al. Endoscopic features of intestinal follicular lymphoma: the value of double-balloon enteroscopy. Endoscopy 39 (Suppl 1): E26-E27, 2007.

9. LeBrun DP, Kamel OW, Cleary ML, Dorfman RF, Warnke RA. Follicular lymphomas of the gastrointestinal tract. Pathologic features in 31 cases and bcl-2 oncogenic protein expression. Am J Pathol 140: 1327-1335, 1992.

10. Damaj G, Verkarre V, Delmer A, et al. Primary follicular lymphoma of the gastrointestinal tract: a study of 25 cases and a literature review. Ann Oncol 14: 623-629, 2003.

11. Kodama T, Ohshima K, Nomura $K$, et al. Lymphomatous polyposis of the gastrointestinal tract, including mantle cell lymphoma, follicular lymphoma and mucosa-associated lymphoid tissue lymphoma. Histopathology 47: 467-478, 2005.

12. Yanai $S$, Nakamura $S$, Takeshita $M$, et al. Translocation $t(14 ; 18) /$ IGH-BCL2 in gastrointestinal follicular lymphoma: correlation with clinicopathologic features in 48 patients. Cancer 117: 24672477, 2011.

13. Zucca E, Roggero E, Bertoni F, Cavalli F. Primary extranodal
non-Hodgkin's lymphomas. Part 1: Gastrointestinal, cutaneous and genitourinary lymphomas. Ann Oncol 8: 727-737, 1997.

14. Rohatiner A, d'Amore F, Coiffier B, et al. Report on a workshop convened to discuss the pathological and staging classifications of gastrointestinal tract lymphoma. Ann Oncol 5: 397-400, 1994.

15. Nakamura S, Aoyagi K, Iwanaga S, et al. Synchronous and metachronous primary gastric lymphoma and adenocarcinoma: a clinicopathological study of 12 patients. Cancer 79: 1077-1085, 1977.

16. Noda T, Akashi H, Matsueda S, et al. Collision of malignant lymphoma and multiple early adenocarcinomas of the stomach. Arch Pathol Lab Med 113: 419-422, 1989.

17. Hamaloglu E, Topaloglu S, Ozdemir A, Ozenc A. Synchronous and metachronous occurrence of gastric adenocarcinoma and gastric lymphoma: a review of the literature. World J Gastroenterol 12: 3564-3574, 2006.

18. Suarez F, Lortholary O, Hermine $O$, Lecuit M. Infectionassociated lymphomas derived from marginal zone B cells: a model of antigen-driven lymphoproliferation. Blood 107: 30343044, 2006.

19. Sakai T, Ogura Y, Narita J, et al. Simultaneous early adenocarcinoma and mucosa-associated lymphoid tissue (MALT) lymphoma of the stomach associated with Helicobacter pylori infection. Gastric Cancer 6: 191-196, 2003.

20. Yamamoto S, Nakase H, Yamashita K, et al. Gastrointestinal follicular lymphoma: review of the literature. J Gastroenterol 45: 370388, 2010.

21. Sentani K, Maeshima AM, Nomoto J, et al. Follicular lymphoma of the duodenum: a clinicopathologic analysis of 26 cases. Jpn J Clin Oncol 38: 547-552, 2008.

22. Sato Y, Ichimura K, Tanaka T, et al. Duodenal follicular lymphomas share common characteristics with mucosa-associated lymphoid tissue lymphomas. J Clin Pathol 61: 377-381, 2008.

23. Tanaka F, Tominaga K, Ochi M, et al. Primary duodenal lymphoma: successful rituximab treatment and evaluation by FDGPET. Hepatogastroenterology 54: 1658-1661, 2007.

24. Higuchi K, Komatsu K, Wakamatsu H, et al. Small intestinal follicular lymphoma with multiple tumor formations diagnosed by double-balloon enteroscopy. Intern Med 46: 705-709, 2007.

25. Kanda M, Ohshima K, Suzumiya J, et al. Follicular lymphoma of the stomach: immunohistochemical and molecular genetic studies. J Gastroenterol 38: 584-587, 2003.

26. Tzankov A, Hittmair A, Müller-Hermelink HK, Rüdiger T, Dirnhofer S. Primary gastric follicular lymphoma with parafollicular monocytoid B-cells and lymphoepithelial lesions, mimicking extranodal marginal zone lymphoma of MALT. Virchows Arch 441: 614-617, 2002.

27. Eshoa C, Perkins S, Kampalath B, Shidham V, Juckett M, Chang CC. Decreased CD10 expression in grade III and in interfollicular infiltrates of follicular lymphomas. Am J Clin Pathol 115: 862867, 2001.

28. Millar EK, Waldron S, Spencer A, Braye S. CD10 positive thyroid marginal zone non-Hodgkin lymphoma. J Clin Pathol 52: 849850, 1999.

29. Ballesteros E, Osborne BM, Matsushima AY. CD5+ low-grade marginal zone B-cell lymphomas with localized presentation. Am J Surg Pathol 22: 201-207, 1998.

30. Takata K, Sato Y, Nakamura N, et al. Duodenal follicular lymphoma lacks AID but expresses BACH2 and has memory B-cell characteristics. Mod Pathol 26: 22-31, 2013.

31. Dogan A, Bagdi E, Munson P, Isaacson PG. CD10 and BCL-6 expression in paraffin sections of normal lymphoid tissue and B-cell lymphomas. Am J Surg Pathol 24: 846-852, 2000.

(C) 2013 The Japanese Society of Internal Medicine http://www.naika.or.jp/imonline/index.html 